\title{
New Era for Renal-Protective Therapy in Type 2 Diabetes: Better Renal Outcomes in Patients with Type 2 Diabetes Taking Sodium-Glucose Cotransporter 2 Inhibitors versus Dipeptidy1 Peptidase-4 Inhibitors
}

\section{Chan-Hee Jung}

Division of Endocrinology and Metabolism, Department of Internal Medicine, Soonchunhyang University Bucheon Hospital, Soonchunhyang University College of Medicine, Bucheon, Korea

Diabetes is the most common cause of chronic kidney disease (CKD) globally. Diabetes and CKD commonly coexist and are associated with a high risk for cardiovascular (CV) morbidity and mortality. Diabetic kidney disease (DKD) is very prevalent, and the proportion of Korean adults $>30$ years of age with diabetes who had either albuminuria or CKD was found to be over $30 \%$ [1]. The global burden of DKD or DM-induced end-stage renal disease (ESRD) has continued unabated even though allcause mortality and CV mortality are generally declining. Until recently, the only treatment with a demonstrated ability to attenuate DKD was a renin-angiotensin system (RAS) blocker. In addition, residual nephropathy risk remained present in patients with type 2 diabetes mellitus (T2DM) despite multifactorial intensive medical therapy, including antihypertensive agents such as RAS blockers [2]. A progressive decline in renal function in CKD patients has been regarded as an inevitable and unstoppable fact.

Just a few years ago, no class of glucose-lowering agent was considered to be the preferred treatment. Instead, all glucoselowering medication has been considered equal, with a focus on glucose control rather than end-organ protection and comorbidities. More than 22 randomized controlled trials (RCTs) of di-

Received: 31 March 2021, Accepted: 5 April 2021

Corresponding author: Chan-Hee Jung

Division of Endocrinology and Metabolism, Department of Internal Medicine, Soonchunhyang University Bucheon Hospital, Soonchunhyang University College of Medicine, 170 Jomaru-ro, Wonmi-gu, Bucheon 14584, Korea

Tel: +82-32-621-5158, Fax: +82-32-621-5016, E-mail: chanh@schmc.ac.kr peptidyl peptidase-4 (DPP-4) inhibitors, glucagon-like peptide receptor agonists (GLP-1RAs), and sodium-glucose cotransporter 2 (SGLT2) inhibitors on CV or kidney outcomes have been completed, resulting in tremendous evidence that has led to major changes in diabetes care in just 10 years [3]. From several CV trials and dedicated kidney outcome trials, SGLT2 inhibitors have already achieved stardom due to their ability to slow the rate of CKD progression and improve renal outcomes in T2DM. Major endocrinology, nephrology, and cardiology clinical practice guidelines are rapidly updating their recommendations to reflect the emerging evidence that SGLT2 inhibitors provide benefits in terms of CV and kidney protection [4-7]. All current guidelines recommend SGLT2 inhibitors for patients with T2DM who have atherosclerotic cardiovascular disease (ASCVD), CKD, and heart failure (HF). In addition, the European Society of Cardiology and the European Association for the Study of Diabetes guidelines recommend SGLT2 inhibitors independent of metformin [5]. SGLT2 inhibitors are even beginning to be used as the first-line therapy in patients with T2DM who have ASCVD, HF, or CKD.

Three important RCTs assessed the protective effect of DPP4 inhibitors on renal function. The Saxagliptin Assessment of

\section{Copyright $\odot 2021$ Korean Endocrine Society}

This is an Open Access article distributed under the terms of the Creative Commons Attribution Non-Commercial License (https://creativecommons.org/ licenses/by-nc/4.0/) which permits unrestricted non-commercial use, distribution, and reproduction in any medium, provided the original work is properly cited. 
Vascular Outcomes. Recorded in Patients with Diabetes Mellitus-Thrombolysis in Myocardial Infarction 53 (SAVOR-TIMI 53) trial of saxagliptin and the Cardiovascular and Renal Microvascular Outcome Study With Linagliptin (CARMELINA) study of linaglipitin revealed meaningful improvement in albuminuria but no improvement of other renal outcomes, i.e., sustained ESRD, death due to renal failure, or sustained decrease of the estimated glomerular filtration rate (eGFR). The Efficacy, Safety \& Modification of Albuminuria in Type 2 Diabetes Subjects With Renal Disease With LINAgliptin (MARLINA-T2D) trial of linagliptin also failed to show a statistically impressive effect on reducing albuminuria [8]. Thus, in contrast to SGLT-2 inhibitors, DPP-4 inhibitors are not indicated in the latest guidelines as reno-protective agents. As another class of incretin agents, GLP-1RAs also have kidney-related benefits in terms of albuminuria reduction, but they do not have proven benefits for serum creatinine-based kidney outcomes. None of the major trials of DPP-4 inhibitors or GLP-1RAs reported slower rates of decline in the eGFR [9]. On the contrary, CV outcome trials of SGLT2 inhibitors demonstrated that they reduced the risk of serum creatinine-based kidney outcomes and slowed the loss of kidney function across varying levels of eGFR and albuminuria, even in patients who were already receiving RAS blockade. SGLT2 inhibitors reduce kidney events in people with normal albuminuria. Normoalbuminuric DKD is increasingly widespread and T2DM with normal albuminuria is very common; therefore, SGLT2 inhibitors may be an important strategy for kidney risk reduction across the spectrum of diabetes and kidney disease.

Bae et al. [10] compared the renal effects of DPP-4 inhibitors and SGLT2 inhibitors on albuminuria and ESRD in patients with T2DM. They reported that SGLT2 inhibitors were considerably associated with higher absolute risk reductions in all kidney outcomes than were observed for DPP-4 inhibitors. However, unexpectedly, the benefits were statistically insignificant. The authors conducted a network meta-analysis of RCTs due to the presence of few head-to-head trials between the two drugs and the absence of direct comparison studies for the risk of ESRD between SGLT2 inhibitors and DPP-4 inhibitors. In addition, very recent dedicated renal RCTs of SGLT2 inhibitors (Canagliflozin and Renal Events in Diabetes with Established Nephropathy Clinical Evaluation [CREDENCE] and Dapagliflozin and Prevention of Adverse Outcomes in Chronic Kidney Disease [DAPA-CKD]) were not included in this network meta-analysis. A clear benefit for canagliflozin was demonstrated in people with T2DM and high-albuminuric CKD (CRE-
DENCE) and even in subjects without diabetes in a dapagliflozin trial (DAPA-CKD) [11,12]. Furthermore, the MARLINA-T2D trial of linagliptin, which showed a non-significant reduction in albuminuria, was not included in the meta-analysis [8]. Including these RCTs may have demonstrated a significantly superior renal benefit of SGLT2 inhibitors compared to DPP4 inhibitors.

SGLT2 inhibitors have already reached stardom, but are still rising stars in real-world practice for clinicians. Despite the increasing awareness of the benefits of SGLT2 inhibitors, some burdensome barriers make physicians hesitant to use them. In contrast, physicians prescribe DPP-4 inhibitors more easily and widely because they have an excellent side effect profile. The cardio-renal benefits proven by SGLT2 inhibitors can only be achieved if these drugs are prescribed. Several dedicated kidney outcome trials across diverse groups are ongoing. If they show the same results as previous RCTs in terms of renal benefits, SGLT2 inhibitors may have an unrivaled position. Although further direct head-to-head trials are warranted to compare the renal effects of SGLT2 inhibitors and DPP-4 inhibitors, it would also be promising to investigate the additive and synergistic effects of combined therapy with SGLT2 inhibitors and DPP-4 inhibitors on renal benefits.

\section{CONFLICTS OF INTEREST}

No potential conflict of interest relevant to this article was reported.

\section{ORCID}

Chan-Hee Jung https://orcid.org/0000-0001-8988-0187

\section{REFERENCES}

1. Won JC, Lee JH, Kim JH, Kang ES, Won KC, Kim DJ, et al. Diabetes fact sheet in Korea, 2016: an appraisal of current status. Diabetes Metab J 2018;42:415-24.

2. Fioretto P, Dodson PM, Ziegler D, Rosenson RS. Residual microvascular risk in diabetes: unmet needs and future directions. Nat Rev Endocrinol 2010;6:19-25.

3. Giorgino F, Vora J, Fenici P, Solini A. Renoprotection with SGLT2 inhibitors in type 2 diabetes over a spectrum of cardiovascular and renal risk. Cardiovasc Diabetol 2020;19:196.

4. American Diabetes Association. 9. Pharmacologic approaches to glycemic treatment: standards of medical care in 
diabetes-2021. Diabetes Care 2021;44(Suppl 1):S111-24.

5. Cosentino F, Grant PJ, Aboyans V, Bailey CJ, Ceriello A, Delgado V, et al. 2019 ESC guidelines on diabetes, pre-diabetes, and cardiovascular diseases developed in collaboration with the EASD. Eur Heart J 2020;41:255-323.

6. de Boer IH, Caramori ML, Chan JCN, Heerspink HJL, Hurst C, Khunti K, et al. Executive summary of the 2020 KDIGO diabetes management in CKD guideline: evidencebased advances in monitoring and treatment. Kidney Int 2020;98:839-48.

7. Rangaswami J, Bhalla V, de Boer IH, Staruschenko A, Sharp JA, Singh RR, et al. Cardiorenal protection with the newer antidiabetic agents in patients with diabetes and chronic kidney disease: a scientific statement from the American Heart Association. Circulation 2020;142:e265-86.

8. Groop PH, Cooper ME, Perkovic V, Hocher B, Kanasaki K, Haneda M, et al. Linagliptin and its effects on hyperglycaemia and albuminuria in patients with type 2 diabetes and renal dysfunction: the randomized MARLINA-T2D trial. Diabetes Obes Metab 2017;19:1610-9.
9. Thomson SC, Vallon V. Renal effects of incretin-based diabetes therapies: pre-clinical predictions and clinical trial outcomes. Curr Diab Rep 2018;18:28.

10. Bae JH, Park EG, Kim S, Kim SG, Hahn S, Kim NH. Comparative renal effects of dipeptidyl peptidase-4 inhibitors and sodium-glucose cotransporter 2 inhibitors on individual outcomes in patients with type 2 diabetes: a systematic review and network meta-analysis. Endocrinol Metab (Seoul) 2021;36:388-400.

11. Perkovic V, Jardine MJ, Neal B, Bompoint S, Heerspink HJL, Charytan DM, et al. Canagliflozin and renal outcomes in type 2 diabetes and nephropathy. N Engl J Med 2019;380: 2295-306.

12. Wheeler DC, Stefansson BV, Jongs N, Chertow GM, Greene T, Hou FF, et al. Effects of dapagliflozin on major adverse kidney and cardiovascular events in patients with diabetic and non-diabetic chronic kidney disease: a prespecified analysis from the DAPA-CKD trial. Lancet Diabetes Endocrinol 2021;9:22-31. 